\title{
Long-Term Outcome of Anti-Vascular Endothelial Growth Factor Therapy Following Vitrectomy for Submacular and/or Vitreous Hemorrhage in Neovascular Age-Related Macular Degeneration
}

\section{Setsuko Kawakami ( $\square$ setsu@tokyo-med.ac.jp )}

Tokyo Medical University Hospital: Tokyo Ika Daigaku Byoin https://orcid.org/0000-0002-4755-7813

Yoshihiro Wakabayashi

Tokyo Medical University: Tokyo Ika Daigaku

Kazuhiko Umazume

Tokyo Medical University: Tokyo Ika Daigaku

Yoshihiko Usui

Tokyo Medical University: Tokyo Ika Daigaku

\section{Daisuke Muramatsu}

Tokyo Medical University: Tokyo Ika Daigaku

\section{Tsuyoshi Agawa}

Tokyo Medical University: Tokyo Ika Daigaku

\section{Kaori Yamamoto}

Tokyo Medical University: Tokyo Ika Daigaku

\section{Hiroshi Goto}

Tokyo Medical University: Tokyo Ika Daigaku

\section{Research article}

Keywords: age-related macular degeneration, anti-vascular endothelial growth factor therapy, submacular hemorrhage, vitrectomy, vitreous hemorrhage

Posted Date: February 16th, 2021

DOl: https://doi.org/10.21203/rs.3.rs-236894/v1

License: (c) (i) This work is licensed under a Creative Commons Attribution 4.0 International License. Read Full License 


\section{Abstract}

\section{Purpose:}

To study long-term clinical outcomes in patients with submacular hemorrhage (SMH) and/or vitreous hemorrhage $(\mathrm{VH})$ associated with neovascular age-related macular degeneration (nAMD), who received pars plana vitrectomy (PPV) followed by anti-vascular endothelial growth factor (VEGF) therapy.

\section{Methods:}

In this retrospective case series, 25 eyes with $\mathrm{SMH}$ and/or VH associated with nAMD were treated by PPV and followed for at least 24 months. When exudative changes were unresolved or recurred after PPV, additional intravitreal anti-VEGF therapy was given.

\section{Results:}

Mean best-corrected visual acuity (BCVA) of all patients improved significantly at 1, 3, 6, 12, 18 and 24 months $(P<0.01)$ post-PPV and at the final visit $(P<0.05)$. Mean BCVA of 13 eyes with anti-VEGF therapy improved significantly at $1(P<0.05), 3,6,12(P<0.01), 18$ and 24 months $(P<0.05)$, while 12 eyes without anti-VEGF therapy improved at 1,3 and 6 months $(P<0.05)$ only. Average duration from initial PPV to antiVEGF therapy initiation was $7.54 \pm 9.9$ months. Five of 13 eyes (38.5\%) with anti-VEGF therapy maintained dry macula for more than 1 year after the last injection.

\section{Conclusions:}

In patients with SMH and VH caused by nAMD, administering intravitreal anti-VEGF therapy when exudative changes are unresolved or recur after PPV maintains improved visual acuity long term.

\section{Purpose}

Anti-vascular endothelial growth factor (VEGF) therapy is the first-line therapy for neovascular age-related macular degeneration (nAMD), and sometimes pars plana vitrectomy (PPV) is required in highly active cases with submacular hemorrhage $(\mathrm{SMH})$ and/or vitreous hemorrhage $(\mathrm{VH})$. While PPV may reduce the activity of nAMD [1, 2], unresolved or recurrent exudative changes may require additional anti-VEGF therapy even after PPV. However, in vitrectomized eyes, the half-life of anti-VEGF agent is shortened [3-5], and there is concern that the effect may be diminished faster compared to that in non-vitrectomized eyes.

The aim of our study was to estimate the prognosis of nAMD with SMH and/or VH necessitating PPV, and to examine appropriate management after PPV. We investigated the long-term outcome and the realworld clinical situation of additional anti-VEGF therapy after PPV.

\section{Methods}


This study was a retrospective case series of consecutive patients with nAMD complicated by SMH and/or VH, who were treated with PPV and followed for at least 24 months after PPV at the Department of Ophthalmology of Tokyo Medical University Hospital between April 2009 and March 2017. The study adhered to the tenets of the Declaration of Helsinki and was approved by the institutional review board. All subjects provided written informed consent for the treatment and for this study. Inclusion criteria were nAMD with thick SMH of at least 1.5 disc diameter involving the fovea or VH. Exclusion criteria were other concurrent intraocular diseases. Intravitreal injection of anti-VEGF agent was performed after PPV for unresolved or recurred exudative changes detected by ophthalmoscope or optical coherence tomography (OCT). In some cases, the treat and extend (TAE) regimen $[6,7]$ was used for subsequent injections depending on the activity of nAMD while receiving anti-VEGF therapy.

The patients were examined for best-corrected visual acuity (BCVA), intraocular pressure, slit-lamp biomicroscopy, indirect ophthalmoscopy, OCT examination, and fluorescein and indocyanine green angiography before the initial PPV (baseline). Choroidal neovascularization (CNV) subtypes; namely, polypoidal choroidal vasculopathy (PCV) and retinal angiomatous proliferation (RAP), were diagnosed by fundus examination, OCT, fluorescein and/or indocyanine green angiography as reported previously [813]. However, as hemorrhage often deteriorated image quality, postoperative imaging was performed if preoperative evaluation was not possible. After the initial PPV, the patients were examined every month for BCVA, intraocular pressure, slit-lamp biomicroscopy, indirect ophthalmoscopy and OCT examination. From 6 months or more after PPV, these examinations were performed every 2-4 months in less active cases. In the case of TAE, the examinations were performed as per visit interval.

The surgical procedures were as follows. In all eyes, a standard 3-port 25-gauge PPV was performed and posterior vitreous detachment was created if not already present. At the time of PPV, phacoemulsification, aspiration and intraocular lens implantation (PEA-IOL), injection of $32,000 \mathrm{IU} / 0.2 \mathrm{ml}$ of tissue plasminogen activator (tPA) (Monteplase; Eizai Co, Tokyo, Japan) solution into the subretinal space using a 38-gauge flexible cannula (Alcon), and intravitreal injection of anti-VEGF agents were performed as needed. Fluid-air exchange and tamponade with air, $\mathrm{SF}_{6}$ or silicon oil was also conducted when necessary.

Statistical analysis was performed using MedCalc Statistical Software version 16.2.0 (MedCalc Software Bvba, Ostend, Belgium; https://www.medcalc.org; 2016). Visual acuity was converted to logarithm of the minimal angle resolution (logMAR), and counting fingers, hand motion and light perception were also converted to logMAR as reported previously [14]. Wilcoxon signed-rank test was used for comparison of preoperative and postoperative visual acuity, and Fisher's exact probability test and Mann-Whitney's U test were used to compare two groups with and without anti-VEGF therapy following PPV. $P$ values less than 0.05 were considered to be statistically significant.

\section{Results}


In this study, 25 eyes of 25 patients ( 9 women and 16 men) were included. All patients were followed for at least 24 months (mean $47.0 \pm 20.5$; range 24-96 months) after the initial PPV. The mean age was 76.2 \pm 9.3 (range, 48-89) years at the initial PPV. Eighteen eyes were phakic and 7 eyes were pseudo-phakic at the time of surgery. The subtypes of nAMD were PCV in 18 eyes, RAP in 2 eyes, and unclassified in 5 eyes. The reasons for performing PPV were SMH (8 eyes) and VH (17 eyes) associated with nAMD. The average duration of SMH was $9.3 \pm 4.6$ (range 4-17) days. The average duration of $\mathrm{VH}$ was $45.9 \pm 40.0$ (range, 13-142) days, with the exception of 4 eyes with unknown duration. The average greatest diameter of the SMH was $4.0 \pm 1.6$ (range 2.0-6.7) disc diameters. Thirteen eyes had previously been treated with photocoagulation (PC), photodynamic therapy (PDT) or intravitreal injection of anti-VEGF drugs. Baseline characteristics of the patients are listed in Table 1.

Table 1

Baseline characteristics of the patients

\begin{tabular}{|lc|}
\hline \multicolumn{2}{|l|}{ Characteristics } \\
\hline Number of patients/eyes & $25 / 25$ \\
\hline Gender male/ female & $16 / 9$ \\
\hline Average age (range) years & $76.2 \pm 9.3(48-89)$ \\
\hline Subtype of AMD & $18 / 2 / 5$ \\
\hline PCV /RAP/ unknown & $8 / 17$ \\
\hline Reason of initial vitrectomy & \\
\hline SMH/VH & \\
\hline AMD: age related macular degeneration PCV: polypoidal choroidal vasculopathy \\
\hline RAP: retinal angiomatous proliferation SMH: submacular hemorrhage \\
\hline VH: vitreous hemorrhage \\
\hline
\end{tabular}

Of the 17 eyes with $\mathrm{VH}, \mathrm{SMH}$ was also detected during vitrectomy in 5 eyes. Simultaneous with PPV, the following procedures were performed: PEA-IOL in 17 eyes, injection of tPA into subretinal space in 13 eyes (8 eyes with SMH only, 5 eyes with VH and SMH), intravitreal injection of anti-VEGF agents in 6 eyes (3 eyes with SMH only, one eye with $\mathrm{VH}$ and $\mathrm{SMH}, 2$ eyes with $\mathrm{VH}$ only), and fluid-air exchange with tamponade in 12 eyes (air in 1 eye, $\mathrm{SF}_{6}$ in 9 eyes, and silicon oil in 2 eyes).

Additional treatments after initial PPV in the 25 eyes are shown in Fig. 1. After the initial PPV, 10 eyes received intravitreal injection of anti-VEGF agent. One eye underwent PDT and subsequently received antiVEGF therapy. Four eyes underwent repeat PPV for postoperative macular hole, postoperative proliferative vitreoretinopathy, silicon oil removal, and recurrent VH (1 eye each), 2 of which later received anti-VEGF therapy. Two eyes underwent PC. Eight eyes received no additional treatment. In the patient with recurrent 
VH after the initial PPV, retinal detachment occurred after the second PPV, and the third PPV and scleral buckling were performed. Therefore, a total of 13 eyes were initiated intravitreal anti-VEGF therapy and 12 eyes did not receive intravitreal anti-VEGF therapy. Subsequent analyses were conducted to compare these two groups.

In all patients, the mean BCVA improved significantly at 1, 3, 6, 12, 18, $24(P<0.01)$ months after PPV, and at the final visit $(P<0.05)$ (Fig. 2).

Of the 13 eyes that were given intravitreal injection of anti-VEGF agents, 12 eyes received aflibercept (IVA) and 1 eye received ranibizumab (IVR). There were no significant differences in gender ratio, age, presence of polyps, and baseline BCVA between the eyes with and those without anti-VEGF therapy. Regarding the reason for the initial PPV (SMH or VH), 6 of 8 eyes (75.0\%) with SMH detected before PPV compared with 7 of 17 eyes (41.2\%) with VH were treated with anti-VEGF therapy after vitrectomy, and the rate was apparently higher in eyes with SMH although there was no significant difference.

The mean BCVA of 13 eyes with anti-VEGF therapy improved significantly at $1(P<0.05), 3,6,12(P<$ $0.01), 18$ and $24(P<0.05)$ months. In comparison, the mean BCVA of 12 eyes without anti-VEGF therapy improved significantly at 1,3 and $6(P<0.05)$ months only (Fig. 3).

Of the 13 eyes with anti-VEGF therapy, BCVA improved in 9 eyes $(69.2 \%)$ and worsened in 1 eye (7.7\%) for more than 3 lines (15 letters) on the EDTRS chart at 24 months after PPV. Among the 12 eyes without anti-VEGF therapy, BCVA improved in 7 eyes (58.3\%) and worsened in 1 eye (8.3\%). The causes of vision loss were chronic macular edema in one eye with anti-VEGF therapy and macular atrophy after massive subretinal hemorrhage in one eye without anti-VEGF therapy.

The average duration from the initial PPV to initiation of anti-VEGF therapy was $7.54 \pm 9.9$ (range 1-32) months. The cumulative percentage of anti-VEGF therapy initiation during follow-up is shown in Fig. 4. In the 13 patients who received anti-VEGF therapy, the cumulative percentage of initiation was $53.8 \%$ at 2 months after surgery and $84.6 \%$ at 10 months. Anti-VEGF therapy was given by an as-needed regimen in 8 eyes and by a TAE regimen in 5 eyes ( 2 eyes converted to as-needed regimen). The mean follow-up period from the initiation of anti-VEGF therapy was $32.7 \pm 17.0$ (range $0-63$ ) months, and the average number of anti-VEGF drug injections until the final observation was $6.2 \pm 3.9$ (range, 1-13). Three eyes underwent PDT after PPV. In one eye, PDT was performed before the initiation of anti-VEGF therapy. In this eye, the exudative change was not resolved after PDT, and anti-VEGF therapy was started resulting in dry macula. In the other two eyes, dry macula was not obtained even after the initiation of anti-VEGF therapy, and PDT was performed which achieved dry macula in one eye, but not in the other.

Five of the 13 eyes (38.5\%) that started anti-VEGF therapy after PPV and 12 of 25 eyes (48.0\%) of eyes that did not receive postoperative anti-VEGF therapy maintained dry macula for more than 1 year after the last injection. Eventually, 17 of 25 eyes (68.0\%) with or without anti-VEGF therapy achieved dry macula. 


\section{Discussion}

We evaluated the rate of initiation of anti-VEGF therapy after PPV for SMH and/or VH associated with nAMD. In the case of SMH caused by AMD, the rate of anti-VEGF therapy started after PPV was reported to be $38.6 \%$ for a mean follow-up period of 15.3 (range $3-70$ ) months by Chang et al. [15] and $80.8 \%$ for a mean follow-up period of 6.3 (range 2-13 months) by Plemel et al. [16]. The rate of initiation of antiVEGF therapy varies among studies because the follow-up period and the conditions for starting antiVEGF therapy are different. We initiated anti-VEGF therapy after PPV in 6 of 8 eyes (75.0\%) with nAMDassociated SMH detected before PPV.

Anti-VEGF therapy was started within 2 months after PPV in approximately one-half of the cases studied. Cases in which anti-VEGF therapy was started early after PPV tended to show delayed improvement in exudative change after the operation. The rate of initiating anti-VEGF therapy was considerably low later than 11 months after PPV.

In all patients, there was a significant improvement in mean BCVA compared to baseline during the whole follow-up period. In patients with anti-VEGF therapy after PPV, the improvement in mean BCVA peaked at 3 to 6 months after surgery but remained significantly improved by 24 months. In contrast, in patients without anti-VEGF therapy, the mean BCVA improved significantly only during the first 6 months after PPV, and there was no significant difference thereafter.

Chang et al. [15] reported that patients who received anti-VEGF treatment had significantly improved visual acuity for 6 months after PPV, whereas those who did not receive anti-VEGF therapy improved only up to 3 months after surgery. In patients without anti-VEGF therapy, although vision is improved during the early stage after PPV due to reduced bleeding and exudative changes, visual acuity tends to decline thereafter when scarring and retinal atrophy gradually progress.

There is little evidence in the literature on the effect of anti-VEGF therapy on nAMD in vitrectomized human eyes. Hahn [17] presented a case of successful treatment with aflibercept following single bevacizumab failure in a patient with recurrent choroidal neovascularization following prior macular translocation vitrectomy surgery for AMD. Jung et al. [18] reported a case series of 4 patients with AMD who were previously vitrectomized for macular pucker or macular hole. In their study, treatment with aflibercept was effective in controlling AMD.

Because of faster clearance of anti-VEGF drug from vitrectomized eyes, injection of anti-VEGF drug in vitrectomized eyes is expected to be less efficient than in non-vitrectomized eyes [3-5]. On the other hand, there are also reports that intraocular pharmacokinetic properties of anti-VEGF drug in vitrectomized eyes are similar to those in non-vitrectomized eyes [19].

This study has some limitations. First, the preoperative characteristics of eyes that had previous photocoagulation, PDT and anti-VEGF also affects the visual acuity outcomes as these eyes may have more significant disease or refractory disease hence leading to the vitreous hemorrhage and/or 
submacular hemorrhage. Second, the additional postoperative treatments were variable, including PDT and repeat surgery for other complications. All these variabilities affect the outcome of vision and the timing of initiation of anti-VEGF treatment.

In conclusion, in patients with SMH and/or VH caused by nAMD, initiation of anti-VEGF therapy when exudative changes are unresolved or recur after PPV maintains the improved visual acuity over a long period. Therefore, anti-VEGF therapy may be useful even in vitrectomized eyes.

\section{Declarations}

\section{Ethics approval and consent to participate}

The research was conducted in accordance with the guiding principles of the Declaration of Helsinki and approved by the institutional review board of Tokyo Medical University (No.SH3782). Informed consent to participate was obtained from all individual participants included in this study.

\section{Consent for publication}

Not applicable

\section{Availability of data and material}

The datasets during and/or analyzed during the current study available from the corresponding author on reasonable request.

\section{Competing interests}

The authors declare that they have no competing interests.

\section{Funding}

The authors did not receive support from any organization for the submitted work. No funding was received to assist with the preparation of this manuscript. No funding was received for conducting this study.

\section{Authors' contributions}

SK and YW were involved in study design, data interpretation, and data analysis.

SK, KU, YU, DM, TA and KY were involved in collection and assembly of data.

SK drafted the manuscript. YW and HG were involved in revising the manuscript critically for important intellectual content. All authors revised the manuscript, approved the manuscript to be published, and agreed to be accountable for all aspects of the work in ensuring that questions related to the accuracy or integrity of any part of the work are appropriately investigated and resolved. 


\section{References}

1. Ikeda T, Sawa H, Koizumi K, Yasuhara T, Yamasaki T. Pars plana vitrectomy for regression of choroidal neovascularization with age-related macular degeneration. Acta Ophthalmol Scand. 2000;78(4):460-4.

2. Sakamoto T, Sheu SJ, Arimura N, Sameshima S, Shimura M, Uemura A, Kawano H, Wu TT, Kubota T, Sohma R, Noda Y. Vitrectomy for exudative age-related macular degeneration with vitreous hemorrhage. Retina. 2010;30(6):856-64.

3. Lee SS, Ghosn C, Yu Z, Zacharias LC, Kao H, Lanni C, Abdelfattah N, Kuppermann B, Csaky KG, D'Argenio DZ, Burke JA, Hughes PM, Robinson MR. Vitreous VEGF clearance is increased after vitrectomy. Invest Ophthalmol Vis Sci. 2010;51(4):2135-8.

4. Christoforidis JB, Williams MM, Wang J, Jiang A, Pratt C, Abdel-Rasoul M, Hinkle GH, Knopp MV. Anatomic and pharmacokinetic properties of intravitreal bevacizumab and ranibizumab after vitrectomy and lensectomy. Retina. 2013;33(5):946-52.

5. Niwa Y, Kakinoki M, Sawada T, Wang X, Ohji M. Ranibizumab and aflibercept: Intraocular pharmacokinetics and their effects on aqueous VEGF level in vitrectomized and nonvitrectoized macaque eyes. Invest Ophthalmol Vis Sci. 2015;56(11):6501-5.

6. Engelbert M, Zweifel SA, Freund KB. Long-term follow-up for type 1 (subretinal pigment epithelium) neovascularization using a modified "treat and extend" dosing regimen of intravitreal antivascular endothelial growth factor therapy. Retina. 2010;30(9):1368-75.

7. Engelbert M, Zweifel SA, Freund KB. "Treat and extend" dosing of intravitreal antivascular endothelial growth factor therapy for type 3 neovascularization/retinal angiomatous proliferation. Retina. 2009;29(10):1424-31.

8. Uyama M, Matsubara T, Fukushima I, Matsunaga H, Iwashita K, Nagai Y, Takahashi K. Idiopathic polypoidal choroidal vasculopathy in Japanese patients. Arch Ophthalmol. 1999;117(8):1035-42.

9. Spaide RF, Yannuzzi LA, Slakter JS, Sorenson J, Orlach DA. Indocyanine green videoangiography of idiopathic polypoidal choroidal vasculopathy. Retina. 1995;15(2):100-10.

10. De Salvo G, Vaz-Pereira S, Keane PA, Tufail A, Liew G. Sensitivity and specificity of spectral-domain optical coherence tomography in detecting idiopathic polypoidal choroidal vasculopathy. Am J Ophthalmol. 2014;158(6):1228-38.

11. Yannuzzi LA, Negrão S, lida T, Carvalho C, Rodriguez-Coleman H, Slakter J, Freund KB, Sorenson J, Orlock $\mathrm{D}$, Borodoker N. Retinal angiomatous proliferation in age-related macular degeneration. Retina. 2001;21(5):416-34.

12. Matsumoto $\mathrm{H}$, Sato $\mathrm{T}$, Kishi S. Tomographic features of intraretinal neovascularization in retinal angiomatous. proliferation Retina. 2010;30(3):425-30.

13. Ravera V, Bottoni F, Giani A, Cigada M, Staurenghi G. Retinal angiomatous proliferation diagnosis: A multiimaging approach. Retina. 2016;36(12):2274-81. 
14. Schulze-Bonsel K, Feltgen N, Burau H, Hansen L, Bach M. Visual acuities "hand motion" and "counting fingers" can be quantified with the Freiburg visual acuity test. Invest Ophthalmol Vis Sci. 2006;47(3):1236-40.

15. Chang W, Garg SJ, Maturi R, Hsu J, Sivalingam A, Gupta SA, Regillo CD, Ho AC. Management of thick submacular hemorrhage with subretinal tissue plasminogen activator and pneumatic displacement for age-related macular degeneration. Am J Ophthalmol. 2014;157(6):1250-7.

16. Plemel DJA, Lapere SRJ, Rudnisky CJ, Tennant MTS. Vitrectomy with subretinal tissue plasminogen activator and gas tamponade for subfoveal hemorrhage: Prognostic factors and clinical outcomes. Retina. 2019;39(1):172-9.

17. Hahn P. Successful treatment of neovascular age-related macular degeneration following single bevacizumab failure using aflibercept in a vitrectomized eye. Clin Ophthalmol. 2014;8:2129-31.

18. Jung JJ, Hoang QV, Arain MZ, Chang S. Aflibercept anti-vascular endothelial growth factor therapy in vitrectomized eyes with neovascular age-related macular degeneration. Acta Ophthalmol. 2016;94(3):e249-50.

19. Ahn SJ, Ahn J, Park S, Kim H, Hwang DJ, Park JH, Park JY, Chung JY, Park KH, Woo SJ. Intraocular pharmacokinetics of ranibizumab in vitrectomized versus nonvitrectomized eyes. Invest Ophthalmol Vis Sci. 2014;55(1):567-73.

\section{Figures}




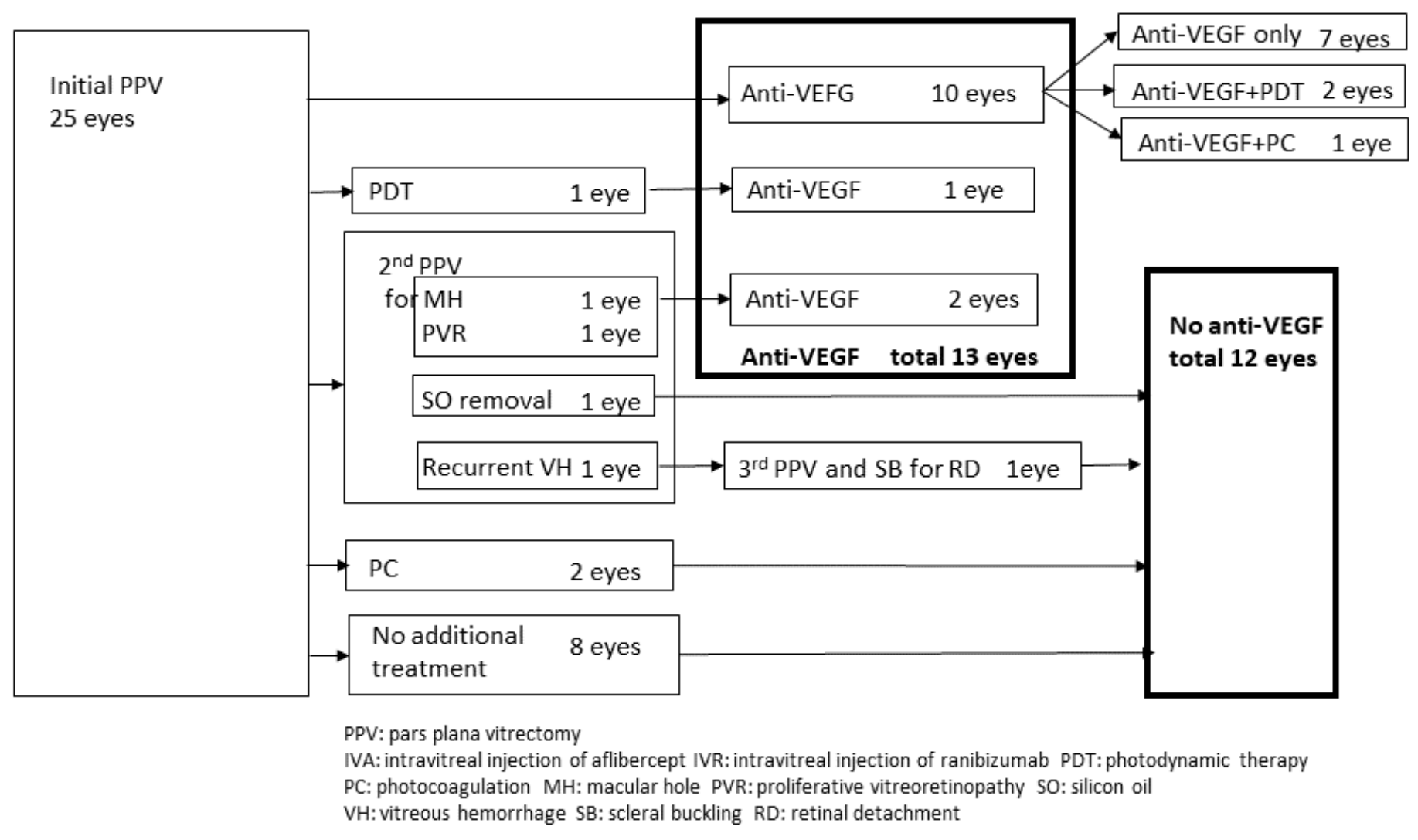

Figure 1

Additional treatments after initial PPV 


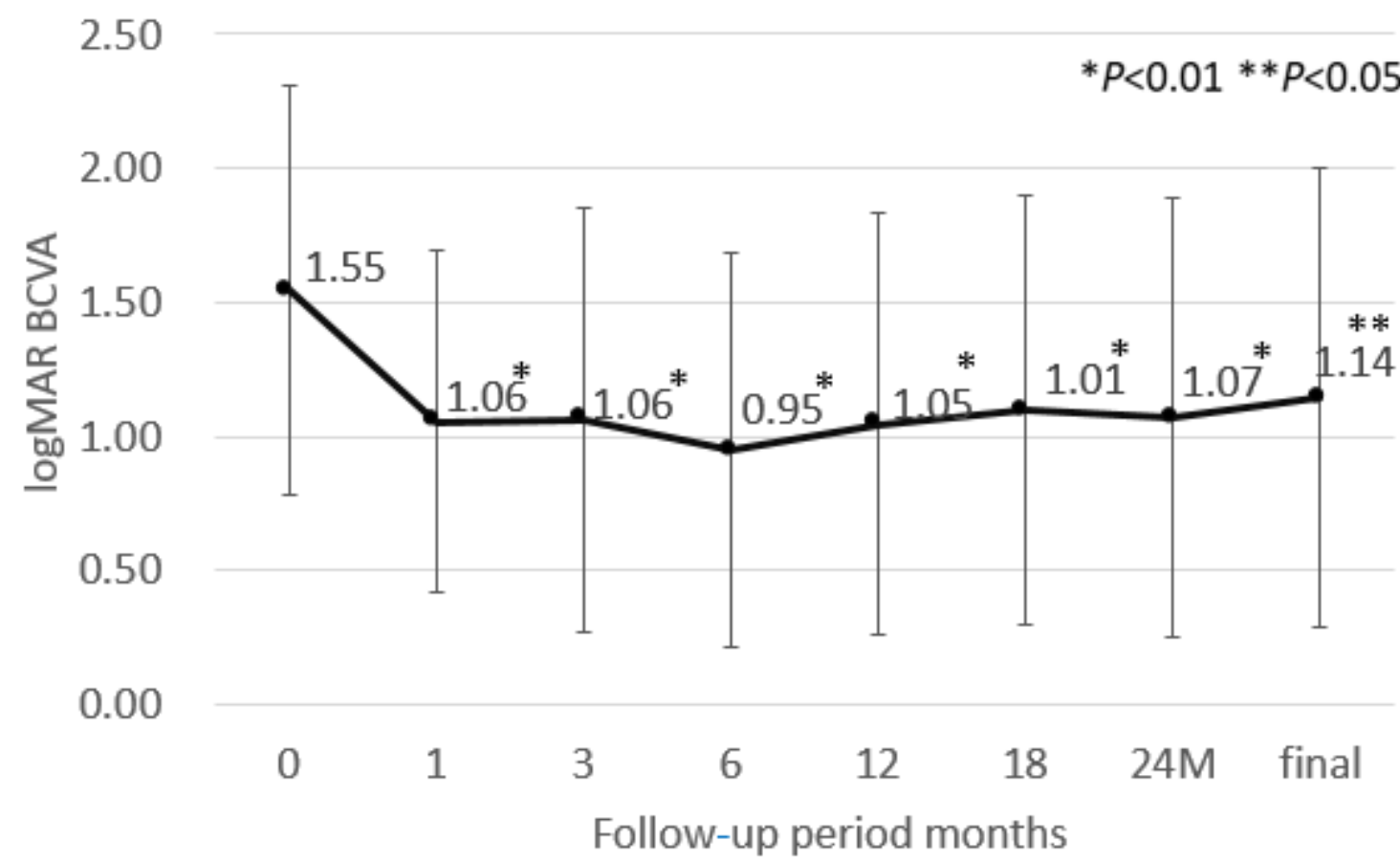

Figure 2

Mean best-corrected visual acuity (BCVA) (logMAR) of all patients. Significant improvement is observed at $1,3,6,12,18$ and 24 months $(P<0.01)$ after vitrectomy and at the final visit $(P<0.05)$

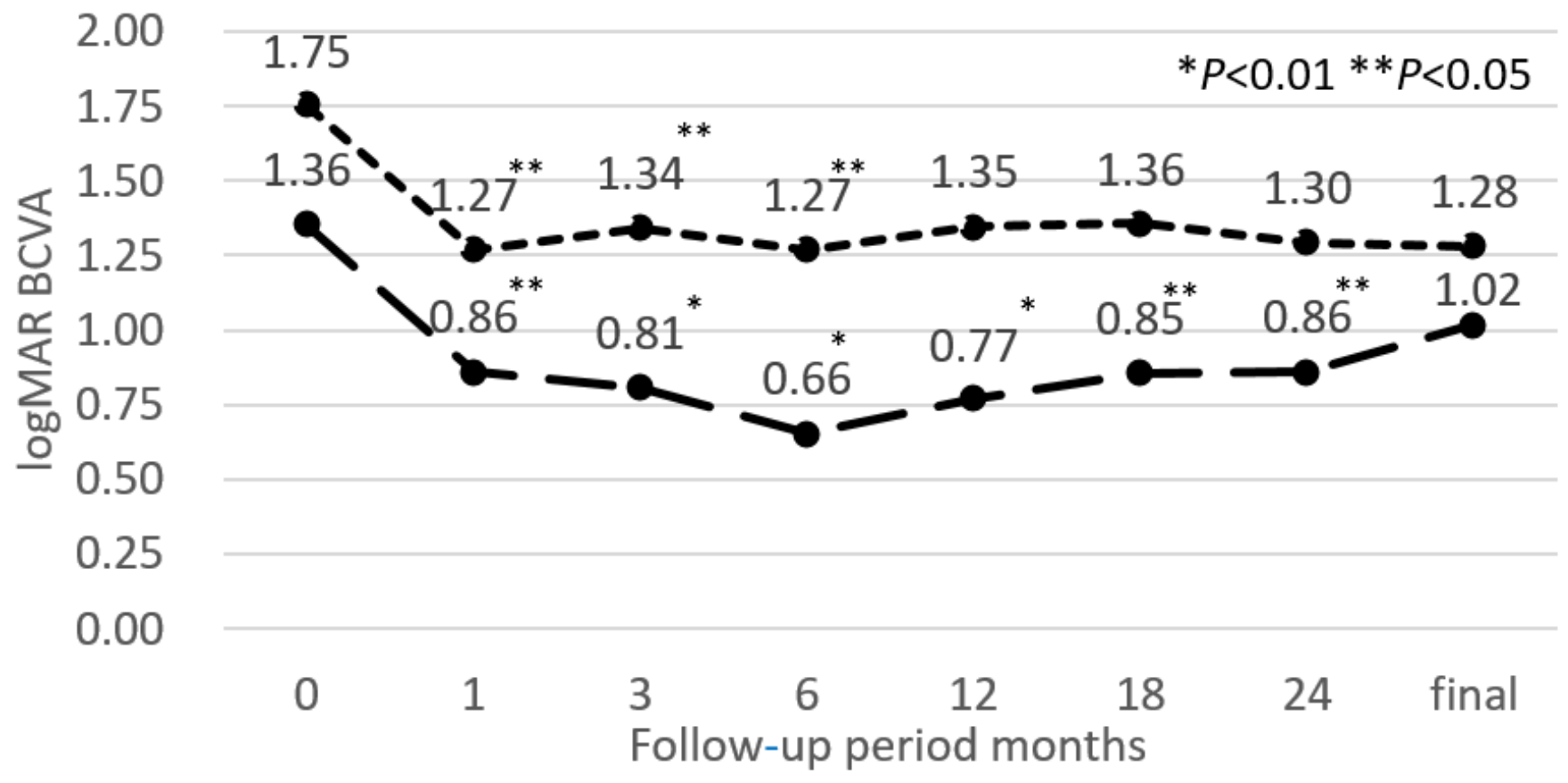

- with anti-VEGF therapy - $\mathbf{- a}$-without anti-VEGF therapy 
Figure 3

Mean best-corrected visual acuity (BCVA) (logMAR) of eyes with and those without anti-VEGF therapy. Significant improvement is observed at $1(P<0.05), 3,6,12(P<0.01), 18$, and $24(P<0.05)$ months in 13 eyes with anti-VEGF therapy, and at 1,3 and 6 months $(P<0.05)$ months in 12 eyes without anti-VEGF therapy



Figure 4

Cumulative percent of initiating anti-VEGF therapy. The rate is $53.8 \%$ at 2 months after surgery and $84.6 \%$ at 10 months 